\title{
A rare case of omental torsion - a surprise diagnosis of acute pelvic pain
}

\author{
Naimah Raza*, Prema Kania, Prashant Bhamare
}

Department of Obstetrics and Gynecology, Bombay Hospital and Medical Research Centre, Mumbai, Maharashtra, India

Received: 22 July 2016

Accepted: 16 August 2016

*Correspondence:

Dr. Naimah Raza,

E-mail: naimahr91@gmail.com

Copyright: (c) the author(s), publisher and licensee Medip Academy. This is an open-access article distributed under the terms of the Creative Commons Attribution Non-Commercial License, which permits unrestricted non-commercial use, distribution, and reproduction in any medium, provided the original work is properly cited.

\begin{abstract}
Omental Torsion is a rare cause of acute abdominal or pelvic pain. It is rarely diagnosed pre-operatively. It poses a great challenge as it closely mimics the other conditions causing acute surgical abdomen, such as appendicitis, ovarian torsion, ruptured ovarian cyst or ectopic pregnancy etc. Hence, often it presents as a surprise intra-operative diagnosis. Though exploratory laparotomy represents the diagnostic and definitive therapeutic procedure, presently Laparoscopy is the first choice procedure.
\end{abstract}

Keywords: Omental torsion, Acute abdomen, Acute pelvic pain, Omentectomy, Omental infarction

\section{INTRODUCTION}

Omental torsion is a condition in which a pedicle of the omental apron twists on its longer axis to such an extent that its vascularity is compromised. Eitel in 1899, first reported a case of omental torsion un-associated with hernia. ${ }^{1}$ Bason and Jones analysed 223 cases of primary torsion and revealed that only one patient had been correctly diagnosed preoperatively. ${ }^{2}$

Primary omental torsion occurs when a mobile, thickened segment of omentum rotates around a proximal fixed point in the absence of any associated or secondary intraabdominal pathology. Secondary omental torsion, on the other hand, occurs due to some underlying pathology, the most common being hernia, cysts, tumours or some foci of abdominal inflammation.

Though the condition can affect any age group, it predominantly affects people between $30-50$ years of age. Male to female preponderance is $2: 1$. Infarction of the greater omentum occurs at the rate of $0.1 \%$ of all laparotomies performed with incidence being 0.0016 $0.37 \% .^{3} 90 \%$ of omental infarction occurs in the right side of the omentum. $0.6-4.8 \%$ of omental infarction is diagnosed preoperatively. ${ }^{3}$

\section{CASE REPORT}

A 26 year old female, married since 3 years, presented with complaints of pain in lower abdomen and pelvic region since 2 days with worsening of pain 2 hours prior to presentation associated with giddiness and weakness. There was no history of fever, vomiting, constipation or any fainting episodes. On enquiry, she gave history of amenorrhea since 48 days. Patient previously had regular cycles with no menstrual complaints. She had undergone one pre-term lower segment caesarean section (at 8 months gestation) one year back in view of pre-term premature rupture of membranes and had a spontaneous abortion after that, at six weeks gestation, six months back. Patient got an intra-uterine contraceptive device (IUCD) inserted six months back after curettage for spontaneous abortion was done. A clinical diagnosis of suspected ruptured ectopic pregnancy or a ruptured ovarian cyst was made.

On examination, the patient was conscious, co-operative and well oriented to time, place and person. Her pulse was $98 / \mathrm{min}$ and her BP was $100 / 60 \mathrm{mmHg}$. There was no pallor. Per abdominal examination revealed tenderness in the pelvic region, more on the right side. There was no guarding or rigidity; the peristaltic sounds were well heard. Per vaginal examination revealed a normal sized 
uterus with right fornicial fullness and bilateral fornicial tenderness, more on the right side.

An urgent urine pregnancy test (UPT) was done which turned out to be Negative. On haemogram, her $\mathrm{Hb}$ was $10.0 \mathrm{~g} / \mathrm{dl}$ and total leucocyte count was 13,300 (raised) with $76 \%$ polymorphs. An Ultrasound was done which showed a minimally bulky and empty uterus with no gestational sac, IUCD in-situ and a small well-walled mass present near the right ovary with increased vascularity and some amount of free fluid in pelvis. The abdomen seemed normal. A differential diagnosis of ectopic pregnancy ruptured ovarian cyst, appendicitis was made and the patient was shifted to the operation theatre.

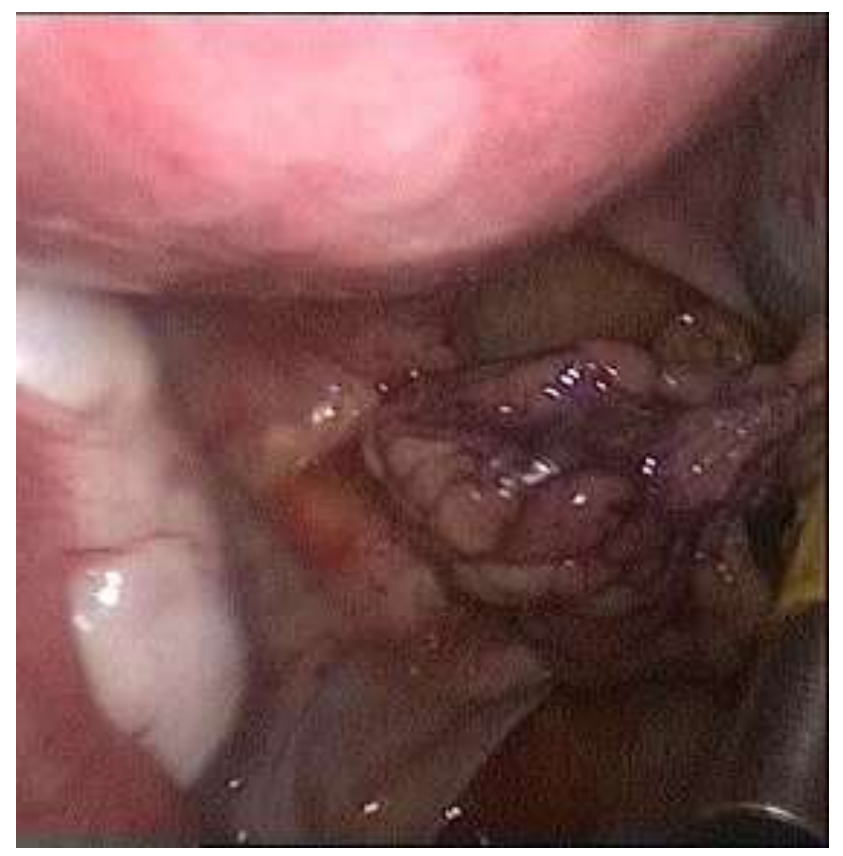

Figure 1: Twisted omental mass adherent to the lateral pelvic wall just below the right ovarian fossa.

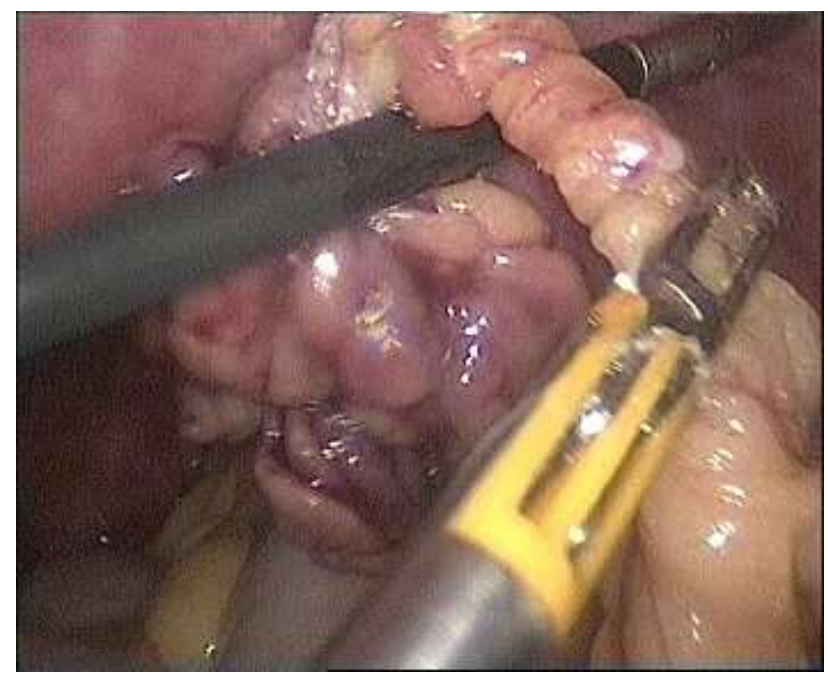

Figure 2: Separated omental mass suspended from its pedicle which has undergone multiple twists.

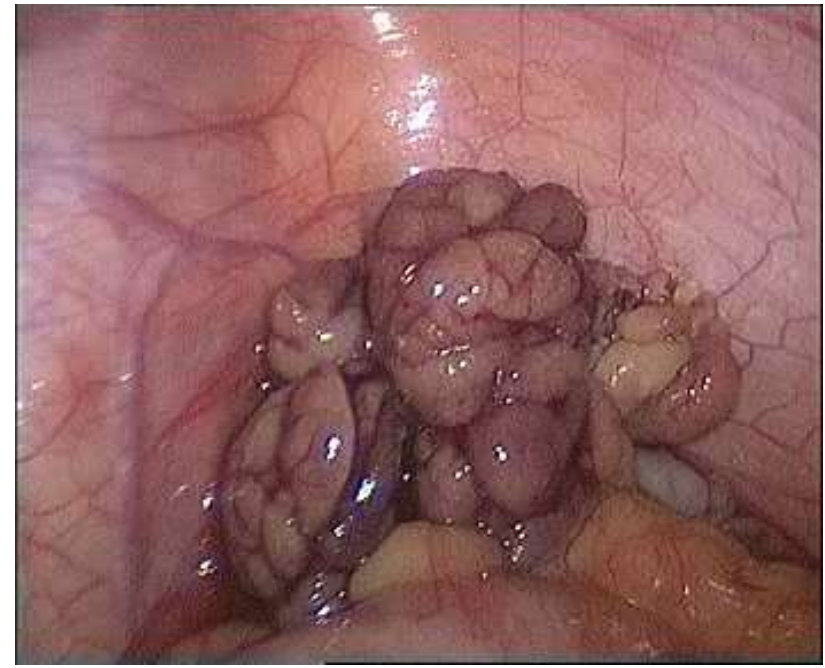

Figure 3: Resected omental mass.

After obtaining adequate consent, under general anaesthesia, a Laparoscopic examination was carried out. It revealed no hemoperitoneum, normal small and large bowel, normal uterus and ovaries and a normal appendix. The omentum was found to be twisted on itself, forming a mass suspended by its pedicle that had undergone multiple twists along its axis. The omental mass lay in the pouch of Douglas, adhered to the pelvic wall just below the right ovarian fossa (Figure 1,2). The omental mass adherent to the pelvic wall was separated and the twisted pedicle of omentum was resected along with it (Figure 3). The specimen was removed and sent for histopathological examination. The patient tolerated the surgery well, had an uneventful recovery and was discharged on the third post-operative day. Histopathology report was suggestive of a $5 \times 7 \mathrm{~cm}$ omental mass with cyanotic look on gross examination. Microscopy was suggestive of congestion, haemorrhage with fat necrosis and small foci of inflammation.

\section{DISCUSSION}

Omental torsion is a rare pathological condition which closely mimics other acute abdominal conditions such as appendicitis and acute diverticulitis or acute pelvic conditions like ruptured ovarian cyst and ovarian torsion. With a history of preceding amenorrhea, a diagnosis of an ectopic pregnancy seemed more probable. The predisposing factors for primary omental torsion include recognized anatomical anomalies like presence of tongue like projections in the omentum, bifid and accessory omentum, anomalous vascular blood supply and other vascular anomalies that modify the weight of omentum, vascular kinking and irregular omental pad seen mostly in obese patients. ${ }^{4}$ Secondary omental torsion is more common and is associated with pre-existing abdominal pathologies including cysts, tumours, foci of intraabdominal inflammations and surgical wounds or scarring and hernial sacs. 5 
The omentum twists in a clockwise direction, with engorgement of the tortuous veins that are easily compressed. This compromises the venous return and the distal omentum becomes congested and oedematous. Recovery may follow or the process may go on. Resultant haemorrhagic extravasation creates a characteristic serosanguinous fluid inside the great omentum and abdominal cavity. As the torsion progresses, arterial occlusion leads to acute haemorrhagic infarction and eventual necrosis of the omentum. ${ }^{6}$ The diagnosis of omental torsion is difficult and is rarely made preoperatively. Ultrasound may usually be normal and might be more helpful is excluding the differential diagnosis. CT scan is more effective in clinching the diagnosis. Many cases are diagnosed after exploratory laparotomy. However, in today's age, Laparoscopy is the first choice modality of treatment, both diagnostic and therapeutic.

Funding: No funding sources Conflict of interest: None declared Ethical approval: Not required

\section{REFERENCES}

1. Eitel GG. Rare omental torsion. NY Med Rec. 1899;55:715-6.

2. Basson SE, Jones PA. Primary torsion of the omentum. Ann R Coll Surg Engl.1981;63:132-4.

3. Concannon ES, Hogan AM, Ryan RS. Primary Omental Infarction: A Rare Cause of Acute Abdominal Pain. Clinical and Experimental Medical Sciences. 2013;1(5):233-40.

4. Young TH, Lee HS, Tang HS. Primary torsion of the greater omentum. Int Surg. 2004;89(2):72-5.

5. Maeda T, Mori H, Cyujo M, Kikuchi N, Hori Y, Takaki H. CT and MR findings of torsion of greater omentum: a case report. Abdom Imaging. 1997;22(1):45-6.

6. Andreuccetti J, Ceribelli C, Manto O, Chiaretti M, Negro P, Tuscano D. Primary omental torsion (POT): a review of literature and case report. World J Emerg Surg. 2011;6:6.

Cite this article as: Raza N, Kania P, Bhamare P. A rare case of omental torsion - a surprise diagnosis of acute pelvic pain. Int J Reprod Contracept Obstet Gynecol 2016;5:xxx-Xx. 